\title{
Heart rate variability and circulating catecholamine concentrations during steady state exercise in healthy volunteers
}

\author{
H-W M Breuer, A Skyschally, R Schulz, C Martin, M Wehr, G Heusch
}

\begin{abstract}
Objectives-To assess whether exercise induced suppression of heart rate variability in the low frequency domain $(0.06-0.15 \mathrm{~Hz})$ is related to the increase in circulating catecholamine concentrations.

Design-Randomised crossover trial of three exercise tests characterised by different workloads. Pharmacological simulation of exercise-induced changes in vagal and sympathetic activity.
\end{abstract}

Participants-Six healthy men with a mean age of $31 \cdot 2$ (SD 3.0) years.

Interventions-Three different workloads of steady state cycling ergometry: control state without cycling, cycling at a target heart rate of 100 beats/min, and cycling at a target heart rate of 150 beats/min. Intravenous infusion of atropine (target heart rate 100 beats $/ \mathrm{min}$ ) followed by the additional infusion of adrenaline and noradrenaline.

Main outcome measures-Fast Fourier analysis of heart rate variability; blood pressure; and venous plasma concentrations of lactate, adrenaline, and noradrenaline.

Results-During the control exercise period there were no changes in the assessed variables compared with the preceding resting period. During exercise at a heart rate of 100 beats/min systolic blood pressure increased and heart rate variability decreased. During exercise at a heart rate of 150 beats/min systolic blood pressure and lactate, adrenaline, and noradrenaline concentrations increased. In addition, low frequency (LF) was lower than during exercise at 100 beats/min, high frequency (HF 0.15-0.80 Hz) resembled that during exercise at 100 beats/min, and diastolic blood pressure was reduced. Infusion of atropine caused no changes in blood pressure or plasma concentrations of lactate, adrenaline, and noradrenaline and decreased heart rate variability. The additional infusion of adrenaline and noradrenaline completely suppressed heart rate variability and increased blood pressure

Conclusions-The reduction in LF and HF during exercise at a heart rate of 100 beats/min, which is not characterised by increased plasma catecholamine concentrations, and during atropine infusion suggests that heart rate variability in the supine state is largely influenced by vagal activity. The additional reduction in LF during exercise at 150 beats/min and during catecholamine infusion may reflect a negative feedback of circulating catecholamines on the sympathetic control of heart rate.

(Br Heart F 1993;70:144-149)

The assessment of heart rate variability has been proposed as a new tool in cardiovascular research. ${ }^{1-3}$ The interest in this method is based on the assumption that the analysis of heart rate variability provides a non-invasive insight into the autonomic regulation of the heart. Therefore heart rate variability is also regarded as a predictor of arrhythmic events in patients after infarction. -6 $^{-6}$

Studies in humans ${ }^{7-9}$ as well as in animals ${ }^{1011}$ have shown relations between sympathetic and parasympathetic activity and the different spectral components of heart rate variability. The power spectral density of the low frequency band (LF) is thought to reflect solely sympathetic activity ${ }^{72}$ or to be an index of both vagal and sympathetic activity..$^{8913}$ Heart rate variability in the high frequency band (HF) is attributed to vagal activity. $^{2814}$ The ratio of LF to HF has been suggested to reflect the sympathovagal balance $^{3}$ or to be an indicator of sympathetic nervous activity. ${ }^{15}$

Attempts have been made to expand the diagnostic use of exercise tests by the additional consideration of heart rate variability. ${ }^{15-17}$ These studies unanimously showed a decrease in heart rate variability during exercise. The well established sympathetic activation which is indicated by an increase in plasma catecholamine concentrations ${ }^{18-20}$ was thus not reflected in an increase in LF power in these studies, as would be anticipated from the association of LF power to sympathetic activity.

The present study was performed to assess the relation between the exercise induced suppression of LF variability and the increase in plasma catecholamine concentrations. We therefore simultaneously measured heart rate variability and plasma catecholamine concentrations during steady state exercise of different intensity. In an additional protocol the exercise induced changes in vagal and sympathetic activity were pharmacologically simulated by intravenous infusion of atropine and catecholamines and their effects on heart rate variability determined. 
Patients and methods

Six healthy men (aged 31.2 (SD 3.0) years) volunteered for the experiments. The study was approved by the ethical committee of the University of Essen Medical School. Witnessed informed consent was obtained from each individual. The contra indications to exercise tests ${ }^{21} 22$ and potential cardiovascular disorders were excluded by physical examination and from the results of exercise electrocardiography, echocardiography, and 24 hour electrocardiography.

The subjects were studied after an overnight fast that included abstinence from caffeine, alcohol, and tobacco. All exercise tests were performed in an air conditioned $\left(20-22^{\circ} \mathrm{C}\right)$ room. The studies for each individual were performed at the same time between 8 am and 12 noon. Speaking was not permitted and external disturbancés were avoided. Respiration was not controlled.

\section{EXERCISE PROTOCOL}

Each volunteer performed three different steady state exercise tests on an electrically braked bicycle (Ergomed 840; Siemens, Erlangen, Germany), in random sequence within three weeks and with a minimum of two days between the studies. The electrodes for electrocardiogram lead II were attached and a cannula (Venflon 2; Pfrimmer-Viggo, Erlangen, Germany) was inserted into an antecubital vein for blood sampling. Physiological saline was infused to avoid thrombotic occlusion of the cannula and to replace the volume of blood sampled. After completion of these preparations the next 20 minutes served as the adaptation period to the experimental setting. The volunteers lay relaxed with their feet resting on the pedals of the ergometer.

Heart rate variability and plasma adrenaline, noradrenaline and lactate concentrations were determined in three sequential intervals of $256 \mathrm{~s}$ during the following 13 minutes of rest. Blood pressure was measured with a sphygmomanometer and standard cuff at the end of the rest period.

An exercise period of eight minutes then followed. In the control state the subject had his feet on the pedals but did not cycle. In another period he cycled to achieve a steady state heart rate of 100 beats/min from the third to the eighth minute, and in another he cycled to achieve a steady state heart rate of 150 beats/min from the third to the eighth minute. The target heart rate was reached in the first three minutes by increasing the workload. At the end of the eighth minute blood was sampled for determination of plasma adrenaline, noradrenaline, and lactate concentrations. Blood pressure was measured immediately at the end of the exercise.

PHARMACOLOGICAL PROTOCOL

As in the exercise protocol the volunteers lay relaxed with their feet resting on the pedals of the ergometer. One cannula was inserted into the right antecubital vein for blood sampling and another was inserted into the left ante- cubital vein for the continuous infusion of atropine (Atropinsulfat Braun; Braun Melsungen, Melsungen, Germany) and catecholamines (Suprarenin and Arterenol; Hoechst, Frankfurt, Germany). The ratio of noradrenaline to adrenaline concentration for each subject was the same as that previously measured at the eighth minute of exercise with a target heart rate of 150 beats $/ \mathrm{min}$. The catecholamines were diluted in $50 \mathrm{ml}$ physiological saline and infused with an electrical infusion pump (Perfusor ED 2; Braun Melsungen, Melsungen, Germany). Blood pressure was recorded every two minutes by an automatic sphygmomanometer (Tonoprint; Speidel and Keller, Jungingen, Germany). After an adaptation period of 20 minutes heart rate variability was determined during the subsequent 13 minutes of rest. Then atropine was infused at a dose sufficient to increase heart rate to 100 beats/min within five minutes with an initial bolus injection ( $1 \mathrm{mg}$ ) and a subsequent continuous infusion (34 (13) $\mu \mathrm{g} / \mathrm{min}$ ). Heart rate variability was determined over five minutes at a steady state heart rate of 100 beats $/ \mathrm{min}$. The catecholamine infusion was then added while the atropine infusion was continued. The infusion rate was increased until the diastolic blood pressure had increased by $30 \mathrm{~mm} \mathrm{Hg}$ or had reached $120 \mathrm{~mm} \mathrm{Hg}$ or the systolic blood pressure had increased by $70 \mathrm{~mm} \mathrm{Hg}$ or had reached $200 \mathrm{~mm} \mathrm{Hg}$. The infusion rate (adrenaline $2.3(1.4) \mu \mathrm{g} / \mathrm{min}$, noradrenaline $14.9(3.7) \mu \mathrm{g} / \mathrm{min})$ was then kept constant for an additional five minutes and heart rate variability assessed. Blood samples were drawn at rest, during the infusion of atropine just before the start of the infusion of catecholamines, and immediately before stopping the infusion of atropine and catecholamines.

DATA ACQUISITION AND ASSESSMENT OF HEART RATE VARIABILITY

The electrocardiogram (lead II) was amplified by a single channel amplifier (Sirecust BS11; Siemens, Erlangen, Germany) and continuously monitored on an oscilloscope. After analog to digital conversion (sampling rate $200 \mathrm{~Hz} / 10$ bit resolution) the electrocardiogram was stored on a floppy disk for later analyses. Instantaneous heart rate was assessed by measuring the time interval between two $R$ waves. An electronic circuit (derivative-threshold-detection algorithm, automatic amplitude adaptation) generated a square wave pulse (duration $140 \mathrm{~ms}$ ) when $75 \%$ of the $\mathrm{R}$ wave amplitude was reached. The time between subsequent pulses was measured using a computer program developed in our laboratory (AT compatible computer $16 \mathrm{MHz}$, numeric coprocessor). The accuracy of this $R R$ interval measurement was tested by an electronically generated signal. The maximal error was found to be less than $2 \mathrm{~ms}$. The measured RR interval length was converted online to heart rate and stored together with a parallel time axis on the hard disk. Before performing further analyses the 
heart rate file was edited visually to exclude potential artefacts, extrasystoles, and postextrasystolic beats. All calculations were based on this corrected data file.

\section{FREQUENCY DOMAIN ANALYSIS}

Digital filtering and the calculation of power spectral densities by means of fast Fourier transform require time series with equidistant intervals between two data points. Therefore, the $R \mathbf{R}$ interval series was converted to a time series with data points sampled every 500 $\mathrm{ms.}^{23}$ Before calculation of the power spectrum the equidistantly sampled heart rate signal was filtered by a digital low pass filter $\left(f_{g}=0.02 \mathrm{~Hz}\right)$ to calculate the mean heart rate. Heart rate fluctuations below $0.02 \mathrm{~Hz}$ were removed by subtracting the mean heart rate from the equidistantly sampled heart rate. After passing through a cosine bell type data window segments of $256 \mathrm{~s}$ (512 data points) were transformed to the frequency domain by fast Fourier transformation. The calculated spectrum was divided into separate spectral bands, LF $(0.06-0.15 \mathrm{~Hz})$ and HF $(0.15-0.8 \mathrm{~Hz})$. The power in each spectral band was determined by summing all calculated spectral lines in the corresponding frequency range. The ratio of LF to $\mathrm{HF}$ was calculated.

\section{DETERMINATION OF LACTATE AND}

CATECHOLAMINE CONCENTRATIONS

Lactate was determined from KF/EDTA stabilised blood samples by the LDH/GPT reaction. ${ }^{24}$

Blood samples for catecholamine analysis were centrifuged immediately after withdrawal and the plasma was frozen in liquid nitrogen. All samples were stored at $-70^{\circ} \mathrm{C}$ for later catecholamine determination by high performance liquid chromatography (NovaPak-C18 3.5 × $150 \mathrm{~mm}$ column; Millipore, Eschborn, Germany).

\section{STATISTICS}

Means (SD) are given. The differences between and within the experimental proce- dures were analysed by the non-parametric Friedman test. When significant $(p<0.05)$ differences were detected the Wilcoxon test was applied to compare single mean values.

\section{Results}

EXERCISE PROTOCOL

Heart rate variability and plasma adrenaline, noradrenaline and lactate concentrations were not different during the three sequential resting periods and were therefore averaged. There were also no differences between the different resting periods preceding the three exercise protocols.

During control state without cycling all variables remained unchanged. During the two other exercise protocols heart rate increased from 69 (9) to 101 (4) beats $/ \mathrm{min}$ and from 70 (8) to 157 (5) beats $/ \mathrm{min}$. Systolic blood pressure increased from 118 (9) to 130 (9) $\mathrm{mm} \mathrm{Hg}$ during exercise with a heart rate of 100 beats/min and from 120 (7) to 169 (21) $\mathrm{mm} \mathrm{Hg}$ during exercise with a heart rate of 150 beats $/ \mathrm{min}$. Diastolic blood pressure was not changed during exercise at 100 beats $/ \mathrm{min}$ and decreased from 78 (5) to 65 (4) $\mathrm{mm} \mathrm{Hg}$ during exercise at 150 beats/min. LF and HF decreased from rest to exercise during both exercise protocols targeted at increasing heart rate (fig 1). LF was lowest during the most streneous exercise protocol. The LF to HF ratio decreased from rest to exercise at 150 beats/min (table). Plasma adrenaline, noradrenaline, and lactate concentrations increased from rest to exercise at 150 beats/min (fig 2 ).

\section{PHARMACOLOGICAL PROTOCOL}

The variables measured during the resting period of the pharmacological protocol did not differ from those measured during the resting periods of the exercise protocol. During the infusion of atropine heart rate increased from 67 (6) to 95 (10) beats $/ \mathrm{min}$. Blood pressure remained constant. LF and HF decreased considerably (fig 1).

During the additional infusion of nor-
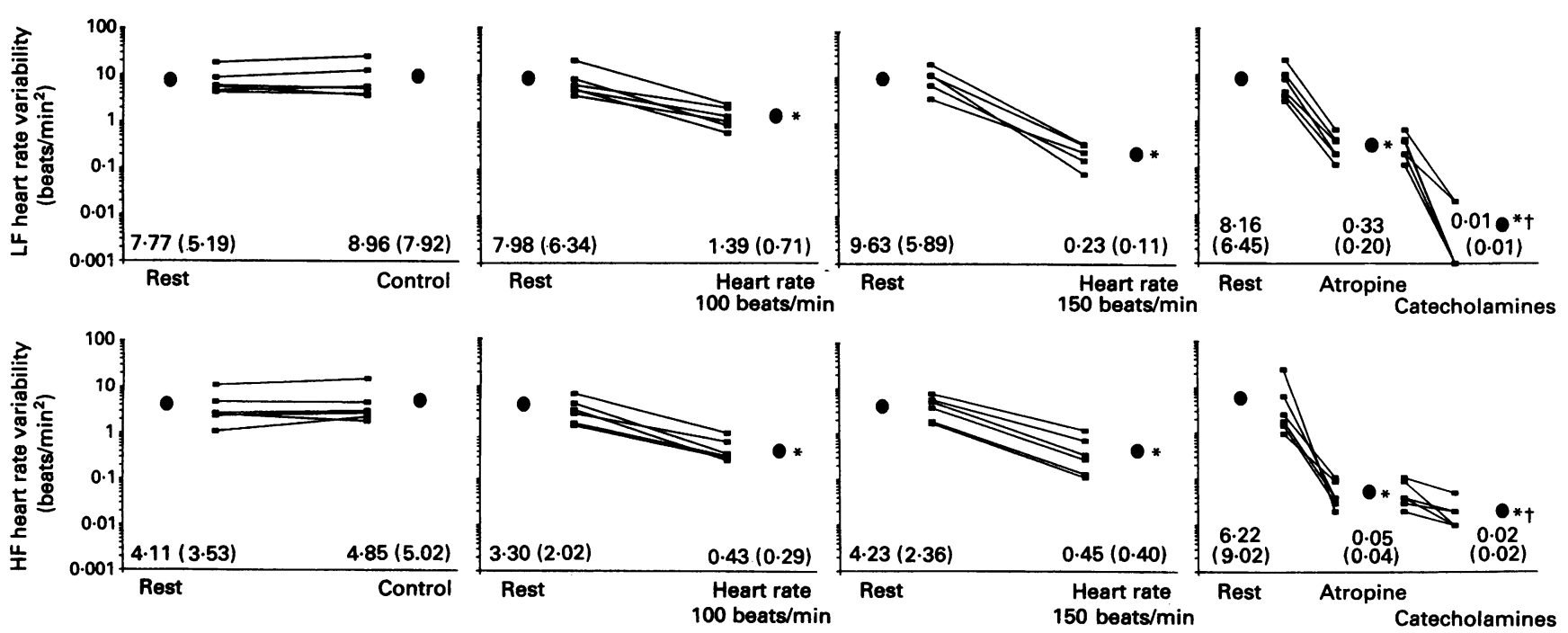

Figure $1 L F$ and HF heart rate variability (in logarithmic units) during exercise and pharmacological protocols. Means (SD) are given in absolute terms $\left({ }^{*} p<0.05\right.$ compared with rest period; $+p<0.05$ compared with atropine infusion). 
Heart rate, systolic and diastolic blood pressures, $L F$ to $H F$ ratio and plasma lactate concentrations during exercise and pharmacological protocols. Values are means (SD)

\begin{tabular}{|c|c|c|c|c|c|}
\hline & \multirow{2}{*}{$\begin{array}{l}\text { Heart rate } \\
\text { (beats } / \mathrm{min} \text { ) }\end{array}$} & \multicolumn{2}{|c|}{ Blood pressure $(m m \mathrm{Hg})$} & \multirow[b]{2}{*}{$L F / H F$} & \multirow{2}{*}{$\begin{array}{l}\text { Lactate } \\
\text { (mmolll) }\end{array}$} \\
\hline & & Systolic & Diastolic & & \\
\hline $\begin{array}{l}\text { Rest } \\
\text { Control } \\
\text { Rest } \\
\text { Heart rạte } 100 \text { beats/min } \\
\text { Rest } \\
\text { Heart rate } 150 \text { beats/min }\end{array}$ & $\begin{array}{r}66(6) \\
64(5) \\
69(9) \\
101(4) \\
70(8) \\
157(5)\end{array}$ & $\begin{array}{l}\text { Exercise proto } \\
113(7) \\
113(8) \\
118(9) \\
130(9)^{\star} \\
120(7) \\
169(21)^{\star} \\
\text { rmacological p }\end{array}$ & $\begin{array}{l}o l \\
78(5) \\
75(6) \\
78(9) \\
77(6) \\
78(5) \\
65(4)^{\star} \\
\text { otocol }\end{array}$ & $\begin{array}{l}2.3(1 \cdot 1) \\
2 \cdot 0(0 \cdot 5) \\
2 \cdot 8(1.9) \\
3.3(2 \cdot 0) \\
2 \cdot 4(1 \cdot 0) \\
0.8(0 \cdot 7)^{\star}\end{array}$ & $\begin{array}{l}0.79(0.10) \\
0.77(0.08) \\
0.79(0.10) \\
1.08(0.23) \\
0.82(0.13) \\
5.86(1.22)^{\star}\end{array}$ \\
\hline $\begin{array}{l}\text { Rest } \\
\text { Atropine } \\
\text { Catecholamines }\end{array}$ & $\begin{array}{l}67(6) \\
95(10)^{\star} \\
123(6)^{\star} \dagger\end{array}$ & $\begin{array}{l}\text { 115(9) } \\
118(4) \\
186(16)^{\star} \dagger\end{array}$ & $\begin{array}{l}070 \text { (7) } \\
83(5) \\
105(7)^{\star}+\end{array}$ & $\begin{array}{l}2.4(1.3) \\
9.0(9.5) \\
0.5(0.8)^{\star} \dagger\end{array}$ & $\begin{array}{l}0.78(0.17) \\
0.71(0.18) \\
1.03(0.41)\end{array}$ \\
\hline
\end{tabular}

${ }^{\star} \mathrm{p}$ < 0.05 compared with rest period. $\mathrm{tp}<0.05$ compared with atropine infusion.

adrenaline and adrenaline heart rate increased further to 123 (6) beats/min. Systolic blood pressure increased from 118 (4) to 186 (16) $\mathrm{mm} \mathrm{Hg}$ and diastolic blood pressure from 83 (5) to 105 (7) $\mathrm{mm} \mathrm{Hg}$. The remaining heart rate variability was completely suppressed (fig 1 ). In four volunteers plasma lactate concentration increased from 0.79 $(0.22)$ to $1.21(0.38) \mathrm{mmol} / \mathrm{l}$ during the catecholamine infusion. In these volunteers the infusion rate of adrenaline $(2.9(1.3) \mu \mathrm{g} / \mathrm{min})$ was higher than in those volunteers who had no increase in plasma lactate concentrations $(1 \cdot 1(0 \cdot 8) \mu \mathrm{g} / \mathrm{min})$.

\section{Discussion}

Our results confirm the well known decrease in heart rate variability induced by exercise. ${ }^{161725}$ Humoral factors such as circulating catecholamines may thus have a more dominant role than direct neural input in sustaining the tachycardia during steady state exercise. ${ }^{26}$ To study the impact of circulating catecholamines on heart rate variability we therefore measured simultaneously heart rate variability and plasma catecholamine concentrations during different exercise intensities as well as the effect of intravenously infused atropine and catecholamines.
To achieve comparable conditions for all volunteers the workload was adjusted individually with respect to the exercise induced heart rate response. During the pharmacological protocol we intended to achieve the same heart rates as in the exercise protocol with a heart rate of 150 beats $/ \mathrm{min}$. Yet the pressor action of the infused catecholamines limited the maximal infusion rate. Thus, the maximal achieved heart rate during the infusion of catecholamines remained lower than during exercise at a heart rate of 150 beats $/ \mathrm{min}$.

As the tachycardia induced by dynamic exercise is mediated both by an initial rapid vagal withdrawal and by a more delayed increase in sympathetic activity at higher levels of workload, ${ }^{27}$ we simulated the vagal withdrawal with atropine before then starting the infusion of catecholamines.

Nevertheless, there were considerable differences between the two experimental procedures (exercise $v$ infusion of atropine and catecholamines). The plasma concentrations of the infused catecholamines necessary to induce the observed haemodynamic changes were higher than those measured during exercise at a heart rate of 150 beats/min, although heart rate remained lower. This discrepancy, previously reported by others, ${ }^{19} 2930$ may be because most of the endogenously released noradrenaline does not reach the circulating blood because of its early inactivation by reuptake into sympathetic nerve terminals. ${ }^{31}$

Our approach of using an individually calculated ratio of noradrenaline to adrenaline concentrations seems to be more appropriate than a fixed arbitrary ratio for all volunteers. Nevertheless, it remains unclear whether a ratio of noradrenaline to adrenaline calculated from venous blood sampled in an antecubital vein reliably reflects what is happening in the affected organs. ${ }^{32}$ The determination of the catecholamine spillover in the coronary sinus effluent will certainly more closely reflect changes in the activity of cardiac sympathetic nerves. Yet, the present approach is
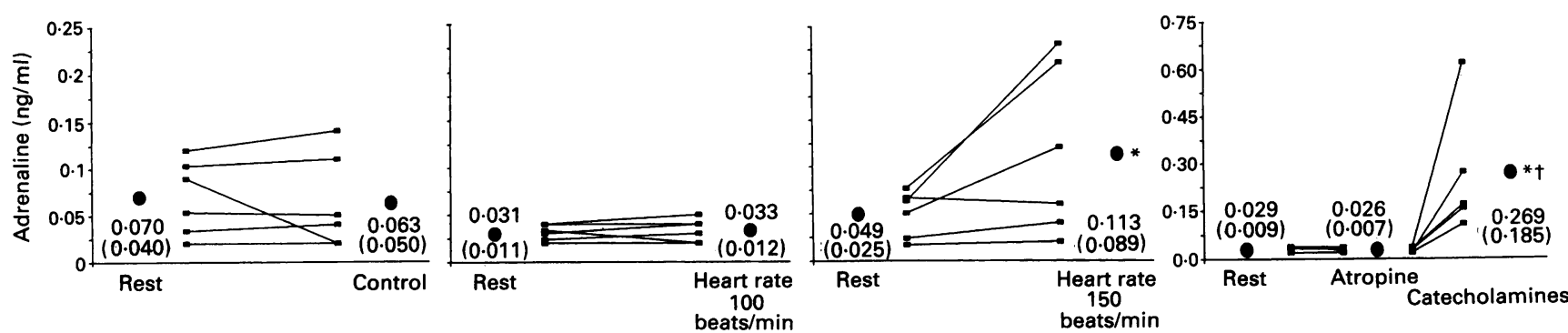

100
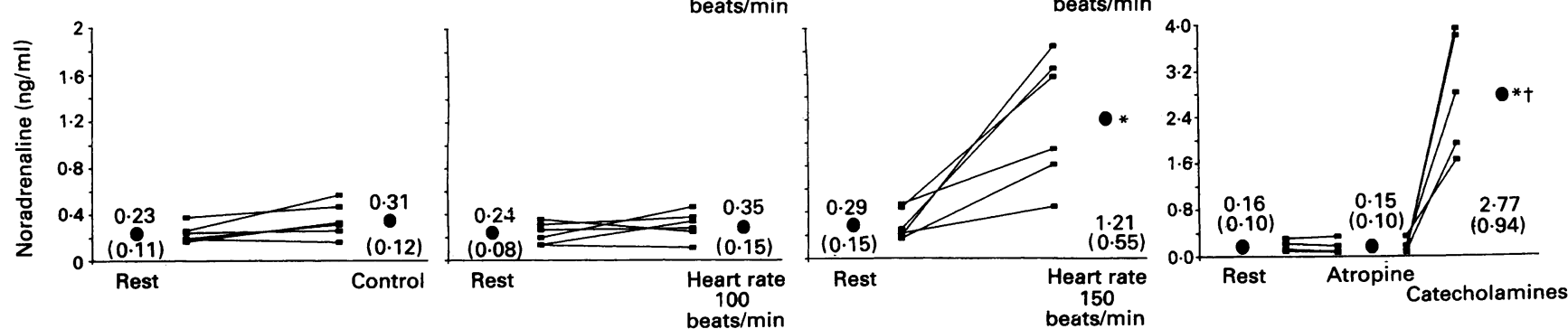

Figure 2 Plasma adrenaline and noradrenaline concentrations with means (SD) during exercise and pharmacological protocols $\left({ }^{*} p<0.05\right.$ compared with rest period; tp $<0.05$ compared with atropine infusion). 
more appropriate to study the feed back between circulating catecholamines originating from the adrenals and other organs and cardiac sympathetic nerve activity.

Heart rate variability in the frequency domain may be assessed by Fourier analysis or by autoregressive techniques. ${ }^{733}$ The calculation of heart rate variability power spectra by autoregressive techniques may improve the spectral resolution but requires predetermination of the autoregressive order of the analysed system. ${ }^{34}$ The following parametric decomposition of a given data set and the calculation of the power spectrum assumes that the selected autoregressive order adequately describes the system under study. This autoregressive order may, however, vary between different subjects and even within a single subject under different physiological conditions. Therefore we calculated the power spectra by fast Fourier transformation because this method is free from any assumptions about the system under study. Despite these methodological differences there is nevertheless a close correlation between heart rate variability indices derived from fast Fourier transformation algorithms and autoregressive methods. ${ }^{35}$ In principle, heart rate variability can be quantified in absolute values or fractional values normalised to total power. Because of the considerable reduction in total power during exercise ${ }^{17}$ small changes in the absolute values of one spectral band can considerably influence the fractional values in all other frequency bands. That is why we studied absolute heart rate variability values.

Our data showing a profound suppression of LF during exercise do not support the concept that LF reflects solely sympathetic activity, ${ }^{712}$ but they do agree with data indicating that LF is influenced by both vagal and sympathetic activity. ${ }^{8913}$ Our data also do not support the assumption that the LF to HF ratio may serve as an index of sympathetic activity when exercise intensity exceeds the ventilatory threshold. ${ }^{15}$ The venous lactate concentration during exercise at 150 beats/min clearly indicates that the ventilatory threshold was indeed exceeded but the LF to HF ratio was nevertheless decreased. ${ }^{36}$ The observed increase in lactate concentration during catecholamine infusion in some volunteers has been previously reported ${ }^{37} 38$ and is explained by the fact that the adrenaline induced decrease in tissue oxygenation of some organs is sufficient to impair normal carbohydrate metabolism so that excess lactate is formed. ${ }^{38}$

Beat by beat modulation of atrioventricular conduction by vagal or sympathetic activity, or both, may contribute to the analysed $R R$ variability. In conscious dogs the oscillations in atrioventricular conduction are predominantly dependent on changes in vagal activity and can be blocked by atropine. Yet, changes in atrioventricular conduction contribute no more than $3 \%$ to changes in the $R R$ interval. ${ }^{39} 40$

The considerable reduction in heart rate variability during exercise at a heart rate of 100 beats/min as well as during atropine infusion point to a major impact of vagal activity on both LF and HF. The additional reduction in LF during exercise at a heart rate of 150 beats/min and of all heart rate variability indices during infusion of catecholamines can be explained by an additional negative feed back of the circulating catecholamines on sympathetic heart rate control. Likewise, a negative relation between spectral and nonspectral measurements of heart rate variability and plasma noradrenaline concentration was recently reported in patients with congestive heart failure. ${ }^{41}$ High plasma noradrenaline concentrations were accompanied by low values of heart rate variability.

The observed reduction in heart rate variability during exercise as well as during the infusion of atropine and catecholamines questions the use of heart rate variability measurements as a tool to differentiate between sympathetic and vagal activity during exercise.

1 Akselrod S, Gordon D, Ubel FA, Shannon DC, Barger AC, Cohen RJ. Power spectrum analysis of heart rate fluctuation: a quantitative probe of beat-to-beat cardiovascular control. Science 1981;213:220-2.

2 Appel ML, Berger RD, Saul JP, Smith JM, Cohen RJ. Beat to beat variability in cardiovascular variables: noise or music? $\mathcal{F} \mathrm{Am}$ Coll Cardiol 1989;14:1139-48.

3 Pagani M, Lombardi F, Guzzetti S, Rimoldi O, Furlan R, Pizzinelli $P$, et al. Power spectral analysis of heart rate and arterial pressure variabilities as marker of sympathovagal interaction in man and conscious dog. Circ Res vagal interaction

4 Farrell TG, Bashir Y, Cripps T, Malik M, Poloniecki J, Bennett ED, et al. Risk stratification for arrhythmic events in postinfarction patients based on heart rate
variability, ambulatory electrocardiographic variables variability, ambulatory electrocardiographic variables and the signal-averaged

5 Myers GA, Martin GJ, Magid NM, Barnett PS, Schaad JW, Weiss JS, et al. Power spectral analysis of heart rate variability in sudden cardiac death: comparison to other methods. IEEE Trans Biomed Eng 1986;33:1149-56.

6 Bigger JT, Jr, Kleiger RE, Fleiss JL, Rolnitzky LM, Steinmann RC, Miller JP, Multicenter Post-infarction Research Group. Components of heart rate variability measured during healing of acute myocardial infarction. Am $₹$ Cardiol 1988;61:208-15.

7 Malliani A, Pagani M, Lombardi F, Cerutti S. Cardiovascular neuronal regulation explored in the frequency domain. Circulation 1991;84:482-92.

8 Pomeranz B, Macaulay RIB, Caudill MA, Kutz I, Adam $\mathrm{D}$, Gordon $\mathrm{D}$, et al. Assessment of autonomic function in humans by heart rate spectral analysis. Am $\mathcal{f}$ Physiol 1985;248:H151-3.

9 Cook JR, Bigger JT, Kleiger RE, Fleiss JL, Steinman RC, Rolnitzky LM. Effect of atenolol and diltiazem on heart period variability in normal persons. $\mathcal{f} \mathrm{Am}$ Coll Cardiol 1991;17:480-4.

10 Lombardi F, Montano N, Finocchiaro ML, Ruscone TG, Contini M, Baselli G. Spectral analysis of heart rate and sympathetic discharge variabilities in decerebrate cats. Circulation 1990;82 (suppl III):III-334 [abstract]

11 Rimoldi O, Pagani M, Pagani MR, Baselli G, Malliani A. Sympathetic activation during treadmill exercise in the conscious dog: assessment with spectral analysis of heart period and systolic pressure variabilities. $f$ Auton Nerv Syst 1990;30:S129-32.

12 Furlan R, Guzzetti S, Crivellaro W, Dassi S, Tinelli M, Baselli G, et al. Continuous 24-hour assessment of the neural regulation of systemic arterial pressure and RR variabilities in ambulant subjects. Circulation 1990;81: v37-47.

13 Kleiger RE, Bigger JT, Bosner MS, Chung M, Cook JR, Rolnitzky LM et al. Stability over time of variables meaRolnitzky LM et al. Stability over time of variables measuring heart rate variabili $1991 ; 68: 626-30$
Cardiol

14 Freeman R Saul JP, Roberts MS, Berger RD, Broadbridge C, Cohen RJ. Spectral analysis of heart rate in diabetic autonomic neuropathy. Arch Neurol 1991;48:185-90.

15 Yamamoto Y, Hughson RL, Peterson JC. Autonomic control of heart rate during exercise studied by heart rate variability spectral analysis. F Appl Physiol 1991 71:1136-42.

16 Arai Y, Saul JP, Albrecht P, Hartley LH, Lilly LS, Cohen 
RJ, et al. Modulation of cardiac autonomic activity during and immediately after exercise. Am $\mathrm{f}$ Physiol 1989;256:H132-41.

17 Perini R, Orizio C, Baselli G, Cerutti S, Veicsteinas A The influence of exercise intensity on the power spectrum of heart rate variability. Eur $\mathfrak{F}$ Appl Physiol trum of heart

18 Christensen NJ, Galbo H. Sympathetic nervous activity during exercise. Annu Rev Physiol 1983;45:139-53.

19 Lehmann M, Rühle K, Schmid P, Klein H, Matthys K, Keul J. Hemodynamic values, plasma catecholamines, and $\beta$-adrenergic receptors on intact polymorphonuclear leukocytes in trained and untrained subjects and patients

20 Perini R, Orizio C, Comande A, Castellano M, Beschi M, Veicsteinas A. Plasma norepinephrine and heart rate dynamics during recovery from submaximal exercise in man. Eur $\mathcal{7}$ Appl Physiol 1989;58:879-83.

21 Schlant RC, Blomqvist CG, Brandenburg RO, DeBurk R, Ellestad MH, Fletcher GF, et al. Guidelines for exercise testing. A Report of the Joint American College of testing. A Report of the Joint American College of committee on Exercise Testing). Circulation 1986;74: committe

22 Löllgen H, Ulmer H-V, Crean P. Recommendations and standard guidelines for exercise testing. Eur Heart $\mathcal{f}$ standard guidelines for

23 Berger RD, Akselrod S, Gordon D, Cohen RJ. An efficient algorithm for spectral analysis of heart rate variability. IEEE Trans Biomed Eng 1986;BME-33:900-

24 Noll F. Bestimmung mit LDH, GPT und NAD. In: Bergmeyer HU, ed. Methoden der enzymatischen Analyse. Weinheim: Verlag Chemie, 1970;1433-7.

25 Bernardi L, Salvucci F, Suardi R, Soldá PL, Calciati A, Perlini S, et al. Evidence for an intrinsic mechanism regulating heart rate variability in the transplanted and the intact heart during submaximal dynamic exercise? Cardiovasc Res 1990;24:969-81.

26 Kamath MV, Fallen EL, McKelvie RS. Effects of steady state exercise on the power spectrum of heart rate variability. Med Sci Sports Exerc 1991;23:428-34.

27 Maciel BC, Gallo L, Neto JAM, Filho ECL, Martins LEB. Autonomic nervous control of the heart rate during dynamic exercise in normal man. Clin Sci 1986; ing dynamic

28 Robinson BF, Epstein SE, Beiser GD, Braunwald E. Control of heart rate by the autonomic nervous system.
Studies in man on the interrelation between baroreceptor mechanisms and exercise. Circ Res 1966;19:400-11.

29 Warren JB, Dalton N. A comparison of the bronchodilator and vasopressor effects of exercise levels of adrenaline in man. Clin Sci 1983;64:475-9.

30 Lake CR, Gullner HG, Polinsky RJ, Ebert MH, Ziegler MG, Bartter FC. Essential hypertension: central and MG, Bartter FC. Essential hypertension: central ar

31 Goldstein DS. Levels of catechols and the clinical assessment of sympathoadrenal activity. In: Ganguly PK, ed ment of sympathoadrenal activity. In: Ganguly PK, ed. Catecholamines

32 Saar N, Bachmann AW, Jackson RV, Gordon RD Different norepinephrine disappearance rate in venous and arterial plasma in man. Clin Exp Hyper-Theory and Practice 1983;31-40 (A5(1)).

33 Sayers BMcA. Analysis of heart rate variability. Ergonomics 1973;16:17-32.

34 Fallen EL, Kamath MV, Ghista DN. Power spectrum of heart rate variability: a non-invasive test of integrated neurocardiac function. Clin Invest Med 1988;11:331-40.

35 Hayano J, Sakakibara Y, Yamada A, Yamada M, Mukai $S$, Fujinami $T$, et al. Accuracy of assessment of cardiac val Ful tone by heart Am $₹$ Cardiol 1991;15:199-204.

36 Robergs RA, Chwalbinska-Moneta J, Mitchell JB, Pascoe DD, Houmard J, Costill DL. Blood lactate threshold DD, Houmard J, Costill DL. Blood lactate threshold Sports Med 1990;11:446-51.

37 Di Salvo RJ, Bloom WL, Brust AA, Ferguson RW, Ferris EB. A comparison of the metabolic and circulatory effects of epinephrine, nor-epinephrine and insulin hypoglycemia with observations on the influence of autonomic blocking agents. $\mathcal{f}$ Clin Invest 1956;35: 568-77.

38 Greene NM. Effect of epinephrine on lactate, pyruvate, and excess lactate production in normal human subjects. F Lab Clin Med 1961;58:682-6.

39 Warner MR, Loeb JM. Beat-by-beat modulation of AV conduction. I. Heart rate and respiratory influences. $A m$ f Physiol 1986;251:H1126-33.

40 Warner MR, De Tarnowsky JM, Whitson CC, Loeb JM Beat-by-beat modulation of AV conduction. II. Autonomic neural mechanisms. Am f Physiol 1986;251: H1134-42.

41 Kienzle MG, Ferguson DW, Birkett CL, Myers GA, Berg WJ, Mariano DJ. Clinical, hemodynamic and sympathetic neural correlates of heart rate variability in con gestive heart failure. Am $\mathcal{F}$ Cardiol 1992;69:761-7. 